\title{
Explosivity Conditions of Aqueous Solutions
}

\author{
R. Thiéry ${ }^{1}$ and L. Mercury ${ }^{2}$
}

Revised version

February, $24^{\text {th }} 2009$

${ }^{1}$ Laboratoire Magmas et Volcans, UMR 6524, CNRS/Clermont Université/OPGC, ClermontFerrand, France.

Address: 5, rue Kessler, F-63038, Clermont-Ferrand Cedex, France.

e-mail: r.thiery@opgc.univ-bpclermont.fr

${ }^{2}$ Institut des Sciences de la Terre d'Orléans, UMR 6113, CNRS/Université d'Orléans, Orléans, France.

Address: 1A, rue de la Férollerie, F-45071, Orléans Cedex, France.

e-mail: lionel.mercury@univ.orleans.fr 


\begin{abstract}
This paper focuses on the conditions of explosive boiling and gas exsolution of aqueous solutions from a thermodynamic point of view. Indeed, the kinetic nature of these processes, hence their explosivity, can be assessed by considering their relation with the spinodal curve of these liquids. First, the concepts of mechanical and diffusion spinodals are briefly recalled, which allows to introduce the notions of superspinodal (explosive) transformations and subspinodal (non-explosive) ones. Then, a quantitative study of spinodal curves is attempted on the binary systems $\mathrm{H}_{2} \mathrm{O}-\mathrm{CO}_{2}$ and $\mathrm{H}_{2} \mathrm{O}-\mathrm{NaCl}$ with equations of state featured by a strong physical basis. It is shown that dissolved gaseous components and electrolytes have an antagonist effect: dissolved volatiles (like $\left.\mathrm{CO}_{2}\right)$ tend to shift the superspinodal region towards lower temperatures, whereas electrolytes (like $\mathrm{NaCl}$ ) tend to extend the metastable field towards higher temperatures.
\end{abstract}

Keywords Metastability, equation of state, spinodal, explosivity, aqueous solution, carbon dioxide, sodium chloride, supersaturation 


\section{Introduction}

Water is the main natural explosive agent on the Earth. This fact is well testified by all forms of volcanic and hydrothermal explosive manifestations, which are produced by a sudden and brutal vaporization of water and other dissolved volatiles from a condensed state, either from aqueous solutions or from supersaturated magmas [1]. This paper is mainly devoted to the first case and tackles the explosivity conditions of aqueous solutions. The traditional approach to such problems is to use kinetic theories of bubble nucleations and growths, and this topic has been already the subject of an abundant literature (see [2-3] and references therein). An alternative and complementary method is applied here by following a phenomenological thermodynamic point of view. Indeed, an explosive situation is generated when a boiling transformation perturbs a liquid up to near or through a thermodynamic frontier, i.e., a spinodal, delimiting a thermodynamically forbidden and unstable region of the phase diagram of the system. In a first part, the theoretical grounds of this paradigm are briefly justified, and it will be shown how boilings and gas exsolutions can be differentiated, depending upon the process conditions, either in explosive transformations or nonexplosive ones. Then, these concepts will be exploited on two important types of aqueous solutions, that are the $\mathrm{H}_{2} \mathrm{O}-\mathrm{CO}_{2}$ and the $\mathrm{H}_{2} \mathrm{O}-\mathrm{NaCl}$ systems, with the help of thermodynamic models founded on solid physical bases. This thermodynamic modeling will permit to decipher the thermodynamic factors controlling the explosivity of boiling and gas exsolution of aqueous solutions.

\section{Explosivity: theoretical concepts}

An explosion is always the response of a system to a physico-chemical perturbation, which has shifted it into a transient and unstable state. The system reacts by a physical or chemical transformation, accompanied by a net volume increase, thus exerting a mechanical work on its surroundings. The damages are mainly due to the elevated kinetic rates, hence to the high rate of 
energy release, characterizing the power or yield of the explosion.

\subsection{Which defines the instability of a system?}

The stability of a system is ruled by the second principle of thermodynamics. A first consequence, which can be derived from this principle, is the mechanical stability criteria:

$$
\left(\frac{\theta)_{V}}{\theta_{V}}\right)_{T} \leq 0
$$

which states that at a constant temperature $T$, an increase of the volume $v$ of a closed system must result to a decrease of the internal pressure $P$. The equality $(\partial \mathrm{P} / \partial v)_{T}=0$ indicates a limiting state between the metastability and the instability fields, defining the so-called mechanical spinodal state. Another consequence of the second principle, which can be derived for fluid mixtures [4], is the diffusion stability criteria, which imposes the spontaneous diffusion (i.e. in the absence of any external force) of species from concentrated regions to less concentrated ones. Mathematically, this statement is formulated by:

$$
\left(\begin{array}{l}
\theta^{2} a^{2} \\
\theta x_{i}^{2}
\end{array}\right)_{T, x_{i}} \geq 0
$$

where $G$ is the Gibbs energy and the $x_{i}$ 's refer to mole fractions. Again, the limiting case $\left(\partial^{2} \mathrm{G} / \partial x^{2}{ }_{i}\right)_{T, P, x_{j}}=0$ corresponds to a frontier of the instability field and defines the so-called diffusion spinodal state [4,5]. Both mechanical and diffusion stability criteria feature the intrinsic stability of a fluid with respect to any density or concentration fluctuations, but are not sufficient to fully characterize the stability of a fluid against the formation of a new phase. This latter aspect can only be assessed by fluid phase equilibria calculations. As a result, the phase diagram (Fig. 1) of any system is subdivided into three domain types: (1) the instability region, where, at least, one stability criteria is violated; (2) the stability region and (3) the metastability fields, where all stability criteria are fulfilled. However, in the metastability region, the fluid phase does not correspond to the most stable state of the system, and the demixion of a new phase is expected to occur, lowering the Gibbs free energy of the system, as predicted by the second principle. The lifetime of a metastable fluid is 
highly variable [6] and depends on kinetic and other external parameters.

Fig. 1 presents these stability, metastability and instability fields for pure water, as calculated by the Wagner and Pruss equation of state [7] in a pressure-temperature plot. Only the mechanical stability criteria is relevant to this one-component system. Limiting stability conditions are encountered along the liquid spinodal curve, noted $\mathrm{Sp}(\mathrm{L})$, and along the gas spinodal curve, noted $\mathrm{Sp}(\mathrm{G})$. Both spinodal curves meet at the critical point CP with the liquid-gas (LG) saturation curve (also called binodal). The gas spinodal curve indicates the theoretical extreme conditions, which can be attained by a metastable gas (referred to as a supercooled gas). In the other way, the liquid spinodal curve marks the furthest theoretical conditions reachable by a metastable liquid (or superheated liquid) before its demixion into a liquid-gas mixture. Fig. 1 depicts also the two main physical processes, which can trigger the boiling of a liquid in a one-component.system: these are (1) isobaric boiling and (2) adiabatic decompression (which can be approximated as a quasiisothermal process for a liquid [1]).

\subsection{Which features the explosivity of a physical transformation?}

In practice, spinodal states of liquid-gas transitions cannot be studied experimentally at the notable exception of the critical point, which is both a gas and liquid spinodal point also touching the stable region. The lifetime of a metastable fluid decreases drastically at the approach of a spinodal curve [6] due to the decreasing work of the vapor formation (= decreasing liquid-vapor surface tension). Thus, only rapid processes, e.g. a very quick heating step or a sudden decompression (Fig. 1), are able to transport a liquid up to spinodal conditions. Energetic barriers of nucleations decrease then to the same magnitude order than molecular fluctuations. Thus, bubble nucleations become active and spontaneous mechanisms, contrasting to the case of weak supersaturation or superheating degrees, where the nucleations of bubbles are known to be a slow process, which must be activated to occur. Kiselev [8], and Kiselev and Ely [9] have calculated precisely the pressure-temperature conditions of this change of nucleation regime for water, introducing the notion of kinetic spinodal. 
This curve mimics the trend of thermodynamic liquid spinodal curve (but is shifted to lower temperatures in a pressure-temperature plot). Moreover, experimental studies of liquid-liquid demixing in alloys or polymers, as well numerical simulations, have demonstrated that the usual matter separation of nucleation-phase growth is replaced by the faster and more efficient process of spinodal decomposition $[2,10]$ in the instability domain, that is when the liquid trespasses the thermodynamic spinodal. Hence, the approach of a superheated liquid up to spinodal conditions is synonym with explosive vaporization.

This paradigm has been validated by the analysis of numerous explosions in the industry. A first type of explosions is caused by the sudden depressurization of liquids. In the specialized literature, this phenomenon is commonly referred to as a BLEVE [11-16] (acronym for a Boiling Liquid Expansion Vapour Explosion). Another type of explosions is produced by the fortuitous contact of a liquid with a hot body at the origin of FCI (Fuel Coolant Interactions) or MFCI (Molten Fuel Coolant Interactions) explosions [17-19]. In each of these categories (BLEVE and FCI), the explosions are interpreted to result mainly from the destabilization of a fluid at near-spinodal conditions. A schematic illustration is given in Fig. 2 in the case of a sudden liquid decompression. The initial state is a liquid at some temperature $T_{0}$ and pressure $P_{0}$, well above the external pressure. The vessel is opened at once, triggering a fast and adiabatic decompression of the liquid. The following depends upon the initial temperature $T_{0}$. In the first case (left part of Fig. 2), the depressurization leads only to some bubble nucleations and produces moderate foaming of the liquid surface. In the second case (right part of Fig. 2), relevant to a BLEVE explosion, the opening of the tank is accompanied by a shock wave, and possibly by its failure with emission of projectiles. The boiling proceeds here by active spontaneous bubble nucleations (when liquid pressure drops below the kinetic spinodal), or conceivably by spinodal decomposition (close to or even beyond the thermodynamic spinodal) in the case of very high depressurization rates. The thermodynamic interpretation [17] of these two different evolutions is given in the bottom part of Fig. 2, and 
involves the spinodal temperature $T_{s p}$ at ambient pressure $\left(T_{s p}=320.45^{\circ} \mathrm{C}=593.6 \mathrm{~K}\right.$ for pure water at one bar, as calculated by the Wagner and Pruss equation of state [1,7]). In the first case, the adiabatic decompression occurs at a temperature below $T_{s p}$ : the depressurization path does not cut the liquid spinodal curve $\mathrm{Sp}(\mathrm{L})$ and no explosion occurs. In the second case, the liquid spinodal curve $\mathrm{Sp}(\mathrm{L})$ is intersected by the adiabatic depressurization path, as the decompression occurs at a temperature above $T_{s p}$, triggering a large-scale explosion. Therefore, we suggest to introduce the terms of subspinodal for non-explosive transformations, and superspinodal for the case of explosive ones.

Note that this interpretation should not be applied too restrictively. Experience shows that some explosive boilings can already occur at temperatures below $T_{s p}[16]$. The spinodal temperature $T_{s p}$ is a pure thermodynamical concept, and the temperature $T_{h n}$ of homogeneous nucleation [1,2] ( $T_{h n}=304^{\circ} \mathrm{C}, 577 \mathrm{~K}$ at one bar for pure water), which is a kinetic parameter, could be more appropriate. Moreover, depending upon the circumstances, a decompression under subspinodal conditions does not always trigger boiling, and the solution becomes then supersaturated. In the case of a transient decompression in a confined system, cavitation (Fig. 1) can take place [1].

Nevertheless, thermodynamics provides us with a simple concept which can help us to analyse the possible evolution, explosive or not, of a boiling or gas exsolution process. However, while the liquid spinodal curve of water is presumably well known, at least in its high-temperature part [5], the topology of spinodal curves of aqueous solutions is poorly known. The purpose of the next two sections is to fill in this gap for $\mathrm{CO}_{2}$ and $\mathrm{NaCl}$ aqueous solutions.

\section{The $\mathrm{H}_{2} \mathrm{O}-\mathrm{CO}_{2}$ system}

The representation of spinodals is a highly demanding task for an equation of state, as calculations are done beyond their fitting range with experimental data. As a consequence, this requires a model with good extrapolation capabilities. The corollary is that we must restrict ourselves to equations of 
state with a good physical basis, and which do not rely on ill-founded empirical correlations. Moreover, the $\mathrm{H}_{2} \mathrm{O}-\mathrm{CO}_{2}$ system involves rather complex molecular interactions, which are not easy to describe rigorously [20,21]: indeed, $\mathrm{H}_{2} \mathrm{O}$ is a strong dipolar molecule, which associates to neighboring water molecules through hydrogen bounds, whereas $\mathrm{CO}_{2}$ is a quadrupole. A first approximation is to use van der Waalslike equations of state (the so-called cubic equations of state), but which incorporate into their attractive $a$ parameter the effects of hydrogen bounds, dipole-dipole and dipole-quadrupole interactions. A first attempt has been made with the Daridon equation of state [22], which is able to represent quantitatively the solubility curves of systems, like $\mathrm{H}_{2} \mathrm{O}-\mathrm{CH}_{4}$, $\mathrm{H}_{2} \mathrm{O}$-hydrocarbons and $\mathrm{H}_{2} \mathrm{O}-\mathrm{CO}_{2}[22,23]$. However, preliminary tests force us to reject this equation of state, as its good description of immiscibility curves has been forced by using complex empirical expressions for the binary interaction parameters $k_{i j}$ between $\mathrm{H}_{2} \mathrm{O}$ and $\mathrm{CO}_{2}$. This leads to a strong deterioration of its extrapolation abilities (in particular, it fails to represent correctly the critical curve of $\mathrm{H}_{2} \mathrm{O}-\mathrm{CO}_{2}$ ). Therefore, we have finally chosen another cubic equation of state, the PengRobinson-Stryjek-Vera (PRSV) equation of state [24-26], which gives good results for mixtures of polar and nonpolar components [27]. A quadratic mixing rule with a zero binary interaction parameter between $\mathrm{H}_{2} \mathrm{O}$ and $\mathrm{CO}_{2}$ has been selected to describe the mixing properties of water and carbon dioxide. Therefore, results given here have to be considered as semi-quantitative. Nevertheless, they should give a reasonable idea of the topology of spinodal curves in water-gases systems. All calculations (thermodynamic properties, binodals, spinodals, critical curves) have been made with the help of the LOTHER library $[20,21,28]$ for fluid phase equilibria modeling.

Fig. 3A gives solubility curves and spinodals calculated by the PRSV equation of state at 323 $\mathrm{K}\left(\approx 50^{\circ} \mathrm{C}\right)$ in a pressure-mole fraction of $\mathrm{CO}_{2}$ diagram. For comparison, the solubility curve $\mathrm{L}(\mathrm{G})$ of $\mathrm{CO}_{2}$ in water, calculated by the more accurate model of Duan and Sun [29], is also drawn. Thus, one can observe that the PRSV equation of state underestimates the $\mathrm{CO}_{2}$ solubility in water, but this was not our first priority. The mechanical spinodal curves for the liquid, noted $\mathrm{mSp}(\mathrm{L})$, and for the 
gas, noted $\mathrm{mSp}(\mathrm{G})$ are plotted too with diffusion spinodal curves $\mathrm{Sp}(\mathrm{L})$ and $\mathrm{Sp}(\mathrm{G})$.

The relations between spinodal curves can be observed more clearly on a molar volume-mole fraction of $\mathrm{CO}_{2}$ (Fig. 3B). Mechanical spinodal curves $\mathrm{mSp}(\mathrm{L})$ and $\mathrm{mSp}(\mathrm{G})$ meet at a pseudocritical point (pCP) where the additional constraint is fulfilled [5,30]:

$$
\left(\frac{\partial^{2} F}{\partial V^{2}}\right)_{T_{N}}=0
$$

The diagram shows also that for the whole composition range, the mechanical instability field (and the pCP) is included in the diffusion instability domain. This result can be generalized and has been demonstrated by Imre and Kraska [5] for different types of mixtures. Thus, for mixtures, the relevant stability criterion is not the mechanical one, but the diffusion one.

Calculations reveal that reversible and adiabatic (i.e. isentropic) liquid decompressions lead to only moderate temperature decreases up to the liquid spinodal curve for pure water [1]. While sudden liquid depressurizations are highly irreversible transformations, they can be assumed also, to a first approximation, as nearly isothermal processes before the onset of the gas demixion. Thus, isothermal pressure-compositions sections (Fig. 3 and 4) provide useful diagrams to analyse the explosive character of these rapid and strong liquid decompressions.

The projections of the $\mathrm{L}(\mathrm{G})$ and $\mathrm{Sp}(\mathrm{L})$ isotherms in a $P-\chi_{\mathrm{CO} 2}$ diagam (Fig. 3A) are almost vertical and parallel. As a consequence, the depressurization of a $\mathrm{CO}_{2}$-supersaturated solution cannot perturb the fluid up to near-spinodal conditions: gas exsolution will always proceed only by moderate bubble nucleations and any decompression process will be subspinodal.

Superspinodal depressurizations are possible in the $\mathrm{H}_{2} \mathrm{O}-\mathrm{CO}_{2}$ system at much higher temperatures. An example is given in Fig. 4, where binodal and spinodal curves at $623 \mathrm{~K}\left(\approx 350^{\circ} \mathrm{C}\right)$ are projected in a $P-x_{\mathrm{CO} 2}$ section. The four curves $L(G), G(L), S p(L)$ and $S p(G)$ join at one critical point $\mathrm{CP}$ of the $\mathrm{H}_{2} \mathrm{O}-\mathrm{CO}_{2}$ critical curve. This figure also shows that a spinodal can be located in the one-phase region in such diagram, for example, the gas-phase spinodal in the liquid phase region. This may appear unusual but makes sense because the spinodals belong to the mother phase on the 
other side of the corresponding branch of the coexistence curve [5]. Moreover, L(G) and Sp(L) curves are not spaced out. For example, a $\mathrm{CO}_{2}$ aqueous solution with $x_{\mathrm{CO} 2}=0.04$ at $350^{\circ} \mathrm{C}$ is saturated at 220 bar, but is already in a spinodal state at 195 bar. Therefore, any brutal decompression of a $\mathrm{CO}_{2}$-saturated solution should lead to a large scale destabilization at this temperature.

Fig. 5 depicts the liquid spinodal curves $\mathrm{Sp}(\mathrm{L})$ in a pressure-temperature diagram for fixed $\mathrm{CO}_{2}$ compositions. The region of negative pressures, which is of interest for describing the capillary properties of $\mathrm{CO}_{2}$ aqueous solutions [31-33], has been also included. Interestingly, it can be noted that spinodal $\mathrm{Sp}(\mathrm{L})$ isopleths present a pressure-temperature trend, which looks similar to the liquid spinodal curve of pure water [1-2,7,31-32]. At low temperatures, the $\mathrm{Sp}(\mathrm{L})$ isopleths are decreasing steeply before to reach a pressure minimum. Then at subcritical temperatures, isopleths are less spaced and sloped, and they finish to meet the $\mathrm{H}_{2} \mathrm{O}-\mathrm{CO}_{2}$ critical curve. The temperature appears as a determining parameter in the explosivity control of $\mathrm{CO}_{2}$ aqueous solutions. The temperature limit can be estimated at ca. $425 \mathrm{~K}, 150^{\circ} \mathrm{C}$ (whose value is to be compared with the spinodal temperature $T_{s p}$ of pure water at 1 bar at $\left.320.45^{\circ} \mathrm{C}[1,7]\right)$. Depressurizations are expected to be subspinodal below this temperature threshold, and superspinodal above. Therefore, the presence of dissolved volatiles in aqueous solutions reduces strongly their metastability field towards lower temperatures and accentuates their explosivity potential with respect to pure water.

The extrapolation of Henry's law constants when aqueous solutions is under negative pressures led to conclude that superheated solutions should have an increasing capability to store dissolved gases with respect to stable solutions [31-32]. This conclusion means that non-saturated

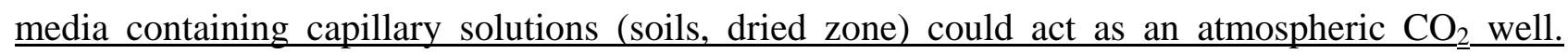
However, what is here demonstrated is that the superheating capability decreases with the $\underline{\text { increasing } \mathrm{CO}_{2}} \underline{\text { mole fraction. As a consequence, certainly there is a subtle equilibrium between the }}$ increasing dissolved gas content in excess of the saturation and the superheating state of the solvent 
which favours it.

\section{The $\mathrm{H}_{2} \mathrm{O}-\mathrm{NaCl}$ system}

The same approach than in the preceding section can be applied to study the explosivity conditions of the $\mathrm{H}_{2} \mathrm{O}-\mathrm{NaCl}$ system. Again, one is faced with the same problem of model selection. The Anderko-Pitzer (AP) equation of state [34] has been chosen here, as it is based on realistic physical hypotheses. It describes $\mathrm{H}_{2} \mathrm{O}-\mathrm{NaCl}$ by means of statistical thermodynamic models [35-36] developed for dipolar hard spheres. This assumption is reasonable at high temperatures, where $\mathrm{NaCl}$ is known to form dipolar ion pairs. However, for this reason, this equation of state is only applicable above $573 \mathrm{~K}, 300^{\circ} \mathrm{C}$. The AP equation of state reproduces quite well the critical curve of $\mathrm{H}_{2} \mathrm{O}-\mathrm{NaCl}$ up to $1100 \mathrm{~K}$ [28, 34], and this another important selection criteria in the search of a good model for spinodal calculations.

Fig. 6 displays a first $P-X_{\mathrm{NaCl}}$ diagram depicting binodal and spinodal isotherms at $623 \mathrm{~K}$, $350^{\circ} \mathrm{C}$. The equation of state reproduces well the tabulated data by Bischoff [37] for the solubility curves $L(G)$ and $G(L)$. The pressures of the diffusion spinodal curves $S p(L)$ and $S p(G)$ decrease with increasing $x_{\mathrm{NaCl}}$ mole fractions. Note also that the spinodal curves run through the stability field of halite at pressures below 100 bars. And others studies [e.g. 31-33] already developed that solid-liquid lines are sensitive to the metastable states inside the system, which should give subtles interplays between all these effects in geochemical contexts.

In terms of $\mathrm{LV}$ partitionning, the $\mathrm{H}_{2} \mathrm{O}-\mathrm{NaCl}$ system presents a dual behaviour concerning its stability during a rapid depressurization. For $x_{\mathrm{NaCl}}$ between 0 and 0.04 , a decompression path down to the atmospheric pressures will intersect the liquid spinodal curve $S p(L)$ : this will trigger an explosive exsolution of $\mathrm{H}_{2} \mathrm{O}$ from the brine, featuring a superspinodal process. Differently, for higher $x_{\mathrm{NaCl}}$, the spinodal curve $\mathrm{Sp}(\mathrm{L})$ runs to negative pressures, and any brutal depressurization can only generate non-explosive boiling of the concentrated brines, characterizing a subspinodal 
transformation.

Two other portrays of the metastability fields of the $\mathrm{H}_{2} \mathrm{O}-\mathrm{NaCl}$ system are given in the $P-\chi_{\mathrm{NaCl}}$ diagrams of Fig. 7A (at $380^{\circ} \mathrm{C}$ ) and Fig. 7B (at $500^{\circ} \mathrm{C}$ ), i.e. at temperatures above the critical point of $\mathrm{H}_{2} \mathrm{O}$. Now, both spinodal $\mathrm{Sp}(\mathrm{L})$ and $\mathrm{Sp}(\mathrm{G})$ isotherms join at a critical point (CP). Another intersection point can be observed at lower pressures, but beware, this is only an artefact generated by the projection of spinodal isotherms onto $P-\chi_{\mathrm{NaCl}}$ planes. Again, $\mathrm{H}_{2} \mathrm{O}-\mathrm{NaCl}$ brines present the same contrasting behaviour, when they are submitted to a sudden depressurization. Below some $x_{\mathrm{NaCl}}$ threshold (e.g. for $x_{\mathrm{NaCl}}<0.08$ at $380^{\circ} \mathrm{C}$ and $x_{\mathrm{NaCl}}<0.19$ at $500^{\circ} \mathrm{C}$ ), fast decompressions will result to superspinodal vaporizations. Thus, subspinodal boilings concern only rather concentrated brines at these elevated temperatures. Note also that the metastability field of supercooled vapours (i.e. between the $G(L)$ and $S p(G)$ curves) extends over a non negligible range of pressures and $\mathrm{NaCl}$ compositions.

Fig. 8 illustrates the metastability fields of the $\mathrm{H}_{2} \mathrm{O}-\mathrm{NaCl}$ system in a $P-T$ diagram by means of a series of spinodal isopleths calculated respectively for $x_{\mathrm{NaCl}}=0.01,0.03,0.05$ and 0.1 . Each isopleth depicts a loop, which is tangential to one point of the $\mathrm{H}_{2} \mathrm{O}-\mathrm{NaCl}$ critical curve. At this critical point, the nature of the isopleth changes from a liquid spinodal $\mathrm{Sp}(\mathrm{L})$ to a gas spinodal $\mathrm{Sp}(\mathrm{G})$. The diagram shows clearly that the addition of $\mathrm{NaCl}$ translates progressively the liquid spinodal curve $\mathrm{Sp}(\mathrm{L})$ to higher temperatures: e.g., the spinodal temperature at 1 bar increases from $320.45^{\circ} \mathrm{C}$ at $x_{\mathrm{NaCl}}=0$ to $403^{\circ} \mathrm{C}$ at $x_{\mathrm{NaCl}}=0.1$ (i.e. $26.5 \mathrm{NaCl}$ wt \%, but in the stability field of halite). Hence, high concentrations of electrolytes favour the metastability of aqueous solutions. This conclusion is in agreement with experimental data of synthetized fluid inclusions of Shmulovich et al. [38] or with the observed behaviour of natural hydrothermal systems involving the circulations of brines [39]. 


\section{$5 \quad$ Conclusion}

Spinodals represent an appealing concept, which allows to link the kinetics of physical transformations to the thermodynamic properties of the system. Explosive vaporizations can be identified as processes, which perturb a liquid up to near-spinodal singularities. This simple criteria, which is commonly applied by safety engineering in the industrial domain, has been generalized here to the case of aqueous solutions. This modeling study shows the antagonist effects of dissolved volatiles and electrolytes (at least, for all components, which behave like $\mathrm{CO}_{2} \underline{\underline{ }}$ and $\mathrm{NaCl}$ ): gaseous species tend to shift the explosivity conditions to lower temperatures, whereas dissolved salts tend to displace spinodal conditions to higher temperatures. This work can give useful indications to constrain the modeling of hydrothermal, phreatic and phreato-magmatic eruptions (for instance, the Lake Nyos disaster, which is explained by some models to result from the explosive $\mathrm{CO}_{2}$ exsolution of dormant supersaturated waters [40]).

\section{Acknowledgement}

This work has been financially supported by the Agence Nationale de la Recherche (ANR) for the project SURCHAUF-JC05-48942.

\section{References}

[1] Thiéry, R., Mercury, L.: Explosive properties of water in volcanic and hydrothermal systems. J. Geophys. Res. (accepted, 2009).

[2] Debenedetti, P.: Metastable Liquids. Princeton University Press, Princeton, New Jersey (1996).

[3] Lasaga, A.: Kinetic theory in the Earth Sciences. Princeton University Press (1998).

[4] Rowlinson, J., Swinton, F.: Liquid and Liquid Mixtures. Butterworth Scientific, 3rd edition, (1982).

[5] Imre, A., Kraska, T.: Stability limits in binary fluid mixtures. J. Chem. Phys. 122, 1-8, 2005. 
[6] El Mekki, M., Ramboz, C., Perdereau, L., Shmulovich, K.I., Mercury, L.: Lifetime of superheated water in a micrometric fluid inclusion. In Rzoska, S.J. and Mazur, V. (Eds), Metastable systems under pressure: Platform for New Technological and Environmental Applications, pp ?-?. Springer, Dordrecht (to be published, 2009).

[7] Wagner, W., Pruss, A.: The IAPWS formulation 1995 for the thermodynamic properties of ordinary water substance for general and scientific use. J. Phys. Chem. Ref. Data 31, 387-535 (2002).

[8] Kiselev, S.: Kinetic boundary of metastable states in superheated and stretched liquids. Physica A 269, 252-268 (1999).

[9] Kiselev, S., Ely, J.: Curvature effect on the physical boundary of metastable states in liquids. Physica A 299, 357-370 (2001).

[10] Debenedetti, P.: Phase separation by nucleation and by spinodal decomposition: fundamentals. In: Kiran, E. et al. (eds), Supercritical Fluids, pp 123-166. Kluwer Academic Publishers, The Netherlands (2000).

[11] Abbasi, T., Abbasi, S.: The boiling liquid expanding vapour explosion (BLEVE): Mechanism, consequence assessment, management. J. Hazardous Materials 141, 480-519 (2007).

[12] Casal, J., Salla, J.: Using liquid superheating for a quick estimation of overpressure in BLEVEs and similar explosions. J. Hazardous Materials A137, 1321-1327 (2006).

[13] Planas-Cuchi, E., Salla, J., Casal, J.: Calculating overpressure from BLEVE explosions. J. Loss Prevention Process Indus. 17, 431-436 (2004).

[14] Pinhasi, G., Ullmann, A., Dayan, A.: 1D plane numerical model for boiling liquid vapor explosion (BLEVE). Int. J.Heat Mass Transfer 50, 4780-4795 (2007).

[15] Salla, J., Demichela, M., Casal., J.: BLEVE: a new approach to the superheat limit temperature. J. Loss Prevention Process Indus. 19, 690-700 (2006).

[16] Reid., R.C.: Possible mechanism for pressurized-liquid tank explosions or BLEVE’s. Science 
203, 1263-1265 (1979).

[17] Reid, R.C.: Superheated liquids. Am. Scientist 64, 146-156 (1976).

[18] Reid, R.C.: Rapid phase transitions from liquid to vapor. Adv. Chem. Engin. 12, 105-208 (1983).

[19] Corradini, M.L., Kim, B.J., Oh, M.D.: Vapor explosions in light water reactors: A review of theory and modeling. Prog. Nuclear Energy 22(1), 1-117 (1988).

[20] Perfetti, E., Thiéry, R., Dubessy, J.: Equation of state taking into account dipolar interactions and association by hydrogen bonding. I- Application to pure water and hydrogen sulfide. Chem. Geol. 251, 58-66 (2008).

[21] Perfetti, E., Thiéry, R., Dubessy, J.: Equation of state taking into account dipolar interactions and association by hydrogen bonding: II- Modelling liquid-vapour equilibria in the $\mathrm{H}_{2} \mathrm{O}-\mathrm{H}_{2} \mathrm{~S}$, $\mathrm{H}_{2} \mathrm{O}-\mathrm{CH}_{4}$ and $\mathrm{H}_{2} \mathrm{O}-\mathrm{CO}_{2}$ systems. Chem. Geol. 251, 50-57 (2008).

[22] Daridon, J., Lagourette, B., Saint-Guirons, H., Xans, P.: A cubic equation of state model for phase equilibrium calculation of alkane + carbon dioxide + water using a group contribution $k_{i j}$. Fluid Phase Equil. 91, 31-54 (1993).

[23] Thiéry, R.: Thermodynamic modeling of aqueous- $\mathrm{CH}_{4}$ bearing fluid inclusions trapped in hydrocarbon-rich environments. Chem. Geol. 227, 154-164 (2006).

[24] Stryjek, R., Vera, J.: An improved Peng-Robinson equation of state with new mixing rules for strongly non ideal mixtures. Can. J. Chem. Eng. 64, 334-340 (1986).

[25] Stryjek, R., Vera, J.: PRSV2: a cubic equation of state for accurate vapour-liquid equilibrium calculations. Can. J. Chem. Eng. 64, 820-826 (1986).

[26] Stryjek, R., Vera, J.: Vapour-liquid equilibria of hydrochloric acid and solutions with the PRSV equation of state. Fluid Phase Equil. 25, 279-290 (1986).

[27] Duan, Z., Hu, J.: A new cubic equation of state and its applications to the modeling of vaporliquid equilibria and volumetric properties of natural fluids. Geochim. Cosmochim. Acta 14, 
2997-3009 (2004).

[28] Thiéry, R.: A new object-oriented library for calculating high-order multivariable derivatives and thermodynamic properties of fluids with equations of state. Comput. Geosciences 22(7), 801-815 (1996).

[29] Duan, Z., Sun, R.: An improved model calculating $\mathrm{CO}_{2}$ solubility in pure water and aqueous $\mathrm{NaCl}$ solutions from 273 to $533 \mathrm{~K}$ and from 0 to 2000 bar. Chem. Geol. 193, 257-271 (2003).

[30] Asselineau, L., Bogdanic, G., Vidal, J.: A versatile algorithm for calculating vapour-liquid equilibria. Fluid Phase Equilibria 3, 273-290 (1979).

[31] Mercury, L., Azaroual, M., Zeyen, H., Tardy, Y.: Thermodynamic properties of solutions in metastable systems under negative or positive pressures. Geochim. Cosmochim. Acta 67, 1769-1785 (2003).

[32] Mercury, L., Pinti, D.L., Zeyen, H.: The effect of the negative pressure of capillary water on atmospheric noble gas solubility in ground water and palaeotemperature reconstruction. Earth Planet. Sci. Lett. 223, 147-161 (2004).

[33] Pettenati, M., Mercury, L., Azaroual, M.: Capillary geochemistry in non-saturated zone of $\underline{\text { soils. Water content and geochemical signatures. Applied Geochem. 23, 3799-3818, } 2008 .}$

[34] Anderko, A., Pitzer, K.: Equation-of-state representation of phase equilibria and volumetric properties of the system NaCl- $\mathrm{H}_{2} \mathrm{O}$ above $573 \mathrm{~K}$. Geochim. Cosmochim. Acta 57, 1657-1680 (1993).

[35] Boublik, T.: Hard sphere equation of state. J. Chem. Phys. 53, 471-472 (1970).

[36] Stell, G., Rasaiah, J., Narang, H.: Thermodynamic perturbation theory for simple polar fluids. J. Mol. Phys. 23, 393-406 (1972).

[37] Bischoff, J.: Densities of liquids and vapors in boiling $\mathrm{NaCl}-\mathrm{H}_{2} \mathrm{O}$ solutions: A PVTX summary from 300 to $500^{\circ} \mathrm{C}$. Am. J. Sci. 291, 369-381 (1991).

[38] Shmulovich, K., Mercury, L., Thiéry, R., Ramboz, C., El Mekki, M.: Superheating ability of 
water and aqueous solutions. Experiments and geochemical consequences. Geochim. Cosmochim. Acta, doi:10.1016/j.gca.2009.02.006 (accepted, 2009).

[39] Orphanidis, E.: Conditions physico-chimiques de précipitation de la barytine épigénétique dans le bassin sud-ouest de la fosse Atlantis II (Mer Rouge): données des inclusions fluides et approche expérimentale. Implications pour le dépôt des métaux de base et métaux précieux. Thèse Université d'Orléans, 180 p (1995).

[40] Rice, A.: Rollover in volcanic crater lakes: a possible cause for Lake Nyos type disasters. J. Volcan. Geotherm. Res. 97, 233-239 (2000).

[41] Tödheide, K., Franck, E.U.: Das Zweiphasengebiet und die kritische Kurve im System Kohlendioxid-Wasser bis zu Drucken von 3500 bar. Z. Phys. Chem. 37, 387-401 (1963). 


\section{Figures}

Fig. 1. Pressure-temperature diagram illustrating the different perturbation processes of liquid water, and their relations with the stable, metastable and unstable fields of $\mathrm{H}_{2} \mathrm{O}$. Solid line: the saturation curve (LG). Dotted lines: the liquid spinodal curve $\mathrm{Sp}(\mathrm{L})$ and the gas spinodal curve $\operatorname{Sp}(G)$.

Fig. 2. a/ Schematic illustration of a subspinodal (left) and a superspinodal decompression (right). b/ Thermodynamic interpretation of the depressurization explosivity in a pressure-temperature diagram.

Fig. 3. a/ Pressure - $\mathrm{CO}_{2}$ mole fraction diagram showing the boundaries of stable, metastable and unstable fields, as calculated by the PRSV equation of state in the $\mathrm{H}_{2} \mathrm{O}-\mathrm{CO}_{2}$ system at $323 \mathrm{~K}$ $\left(\approx 50^{\circ} \mathrm{C}\right)$. Solid lines: the solubility curve $\mathrm{L}(\mathrm{G})$ of $\mathrm{CO}_{2}$ in liquid water, the solubility curve $\mathrm{G}(\mathrm{L})$ of $\mathrm{H}_{2} \mathrm{O}$ in gaseous $\mathrm{CO}_{2}$. Dotted lines: the diffusion liquid $\mathrm{Sp}(\mathrm{L})$ and gas $\mathrm{Sp}(\mathrm{G})$ spinodal curves. Long dashed curves: the mechanical liquid $\mathrm{mSp}(\mathrm{L})$ and gas $\mathrm{mSp}(\mathrm{G})$ spinodal curves. Short dashed curve: solubility curve L(G) calculated by the Duan and Sun model [29]. b/ Molar volume- $\mathrm{CO}_{2}$ mole fraction diagram illustrating the relations between the diffusion and the mechanical metastable fields. Triangle marker: the pseudo-critical point (pCP) at $323 \mathrm{~K}$.

Fig. 4. Projection of the stable, metastable and unstable fields in the $\mathrm{H}_{2} \mathrm{O}-\mathrm{CO}_{2}$ system at $623 \mathrm{~K}$ $\left(\approx 350^{\circ} \mathrm{C}\right)$ onto a pressure- $\mathrm{CO}_{2}$ mole fraction diagram.

Fig. 5. The liquid spinodal curves in a pressure-temperature diagram for the $\mathrm{H}_{2} \mathrm{O}-\mathrm{CO}_{2}$ system, as calculated by the PRSV equation of state. Numbers refer to the mole fraction $x_{\mathrm{CO} 2}$ of dissolved $\mathrm{CO}_{2}$ in the aqueous solution. The filled squares are experimental data of Tödheide and Franck [41] for the $\mathrm{H}_{2} \underline{\mathrm{O}-\mathrm{CO}_{2}}$ critical curve.

Fig. 6. The stable, metastable and unstable fields in a pressure- $\mathrm{NaCl}$ mole fraction diagram for the 
$\mathrm{H}_{2} \mathrm{O}-\mathrm{NaCl}$ system at $623 \mathrm{~K}\left(\approx 350^{\circ} \mathrm{C}\right)$, as calculated by the Anderko and Pitzer equation of state [31]. Solid lines: the solubility curves $L(G)$ and $G(L)$. Dotted lines: the spinodals $S p(L)$ and $S p(G)$. Squared markers: experimental data compiled by Bischoff [34].

Fig. 7. Pressure- $\mathrm{NaCl}$ mole fractions diagrams of the $\mathrm{H}_{2} \mathrm{O}-\mathrm{NaCl}$ diagram, as calculated by the Anderko and Pitzer equation of state [31]. a/ At $653 \mathrm{~K}\left(\approx 380^{\circ} \mathrm{C}\right)$. b/ At $773 \mathrm{~K}\left(\approx 500^{\circ} \mathrm{C}\right)$.

Fig. 8. Stability fields, calculated by the Anderko and Pitzer equation of state [31], of the $\mathrm{H}_{2} \mathrm{O}-\mathrm{NaCl}$ system in a pressure-temperature diagram. Solid lines: the saturation curve (LG) of pure water and the spinodal isopleths of $\mathrm{H}_{2} \mathrm{O}-\mathrm{NaCl}$ fluids (numbers refer to the mole fractions of $\mathrm{NaCl}$ ). Dotted lines: the liquid spinodal curve $\mathrm{Sp}\left(\mathrm{L}, \mathrm{H}_{2} \mathrm{O}\right)$ and gas spinodal curve $\mathrm{Sp}\left(\mathrm{G}, \mathrm{H}_{2} \mathrm{O}\right)$ of pure water. 


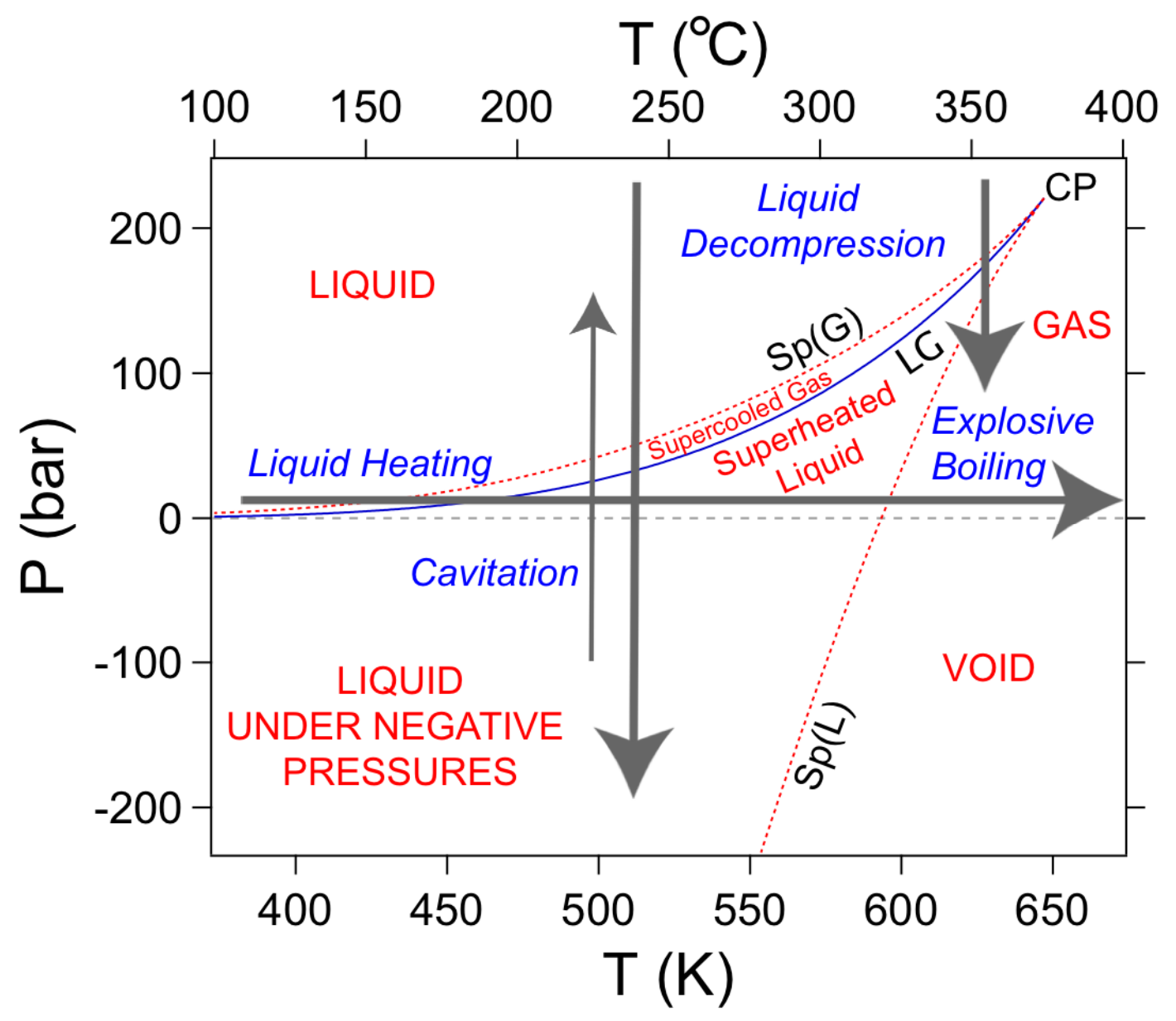

Figure 1 
nitial state:

a pressurized liquid

a/

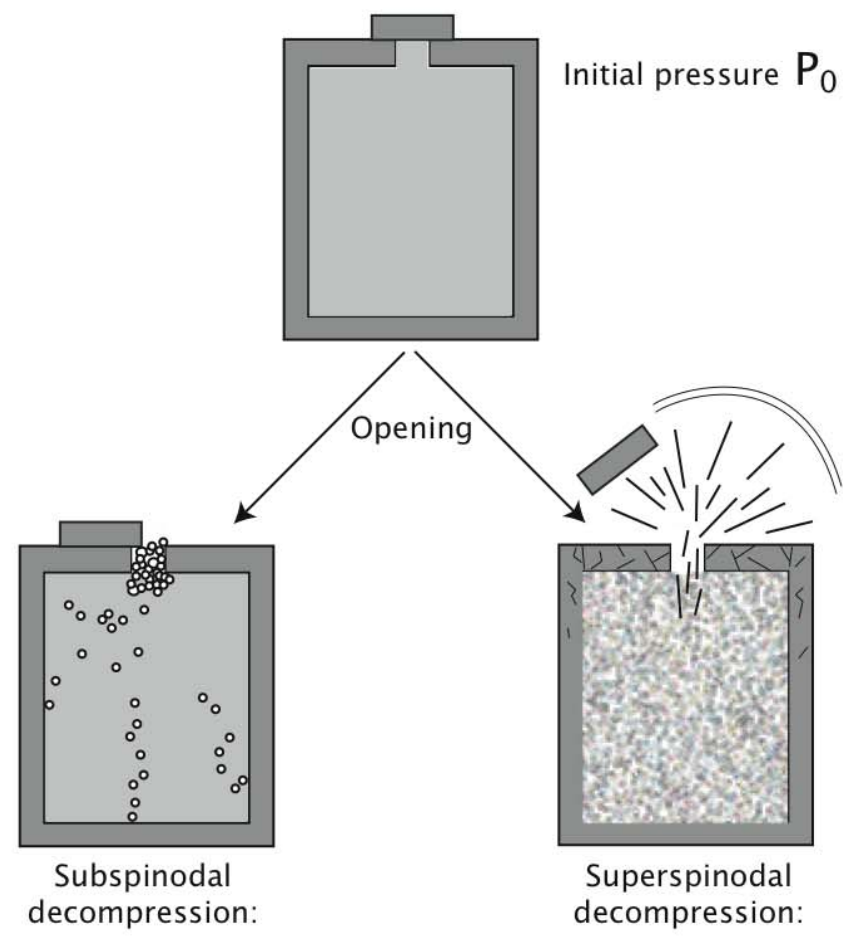

Non-explosive boiling and gas exsolution

Explosive boiling and gas exsolution

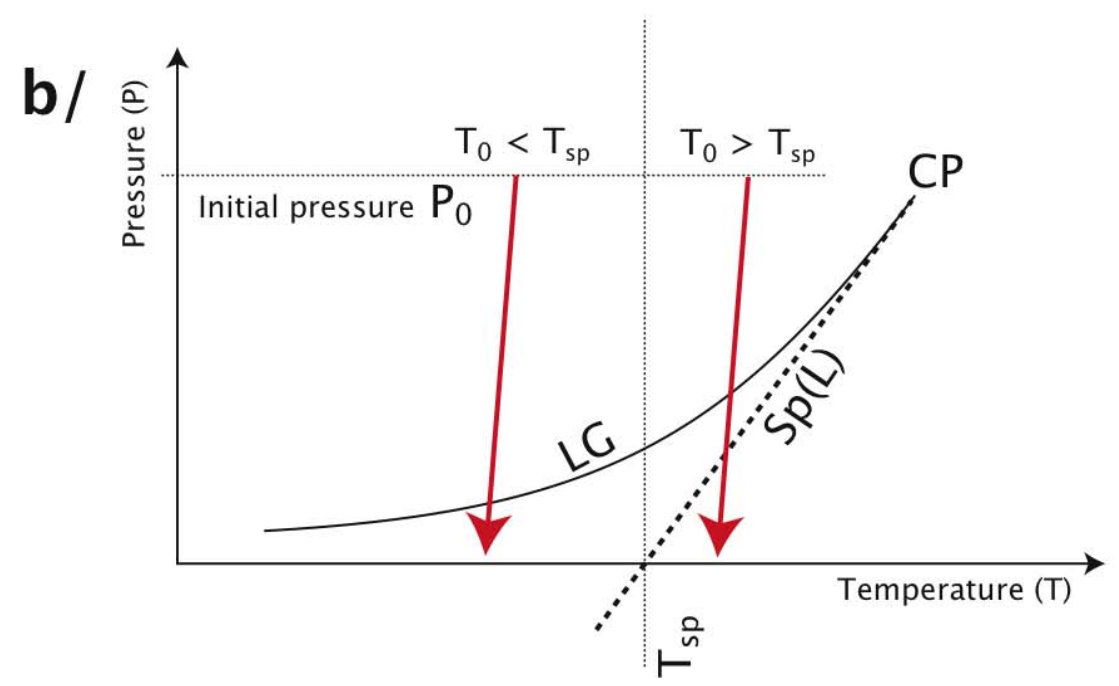

Figure 2 


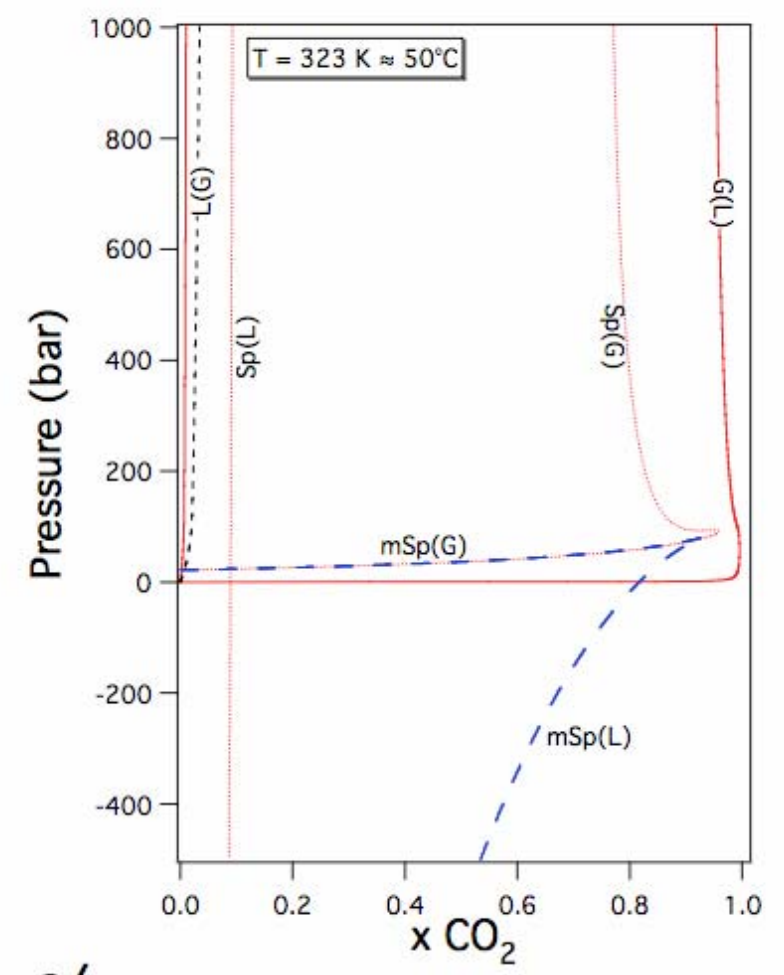

a/

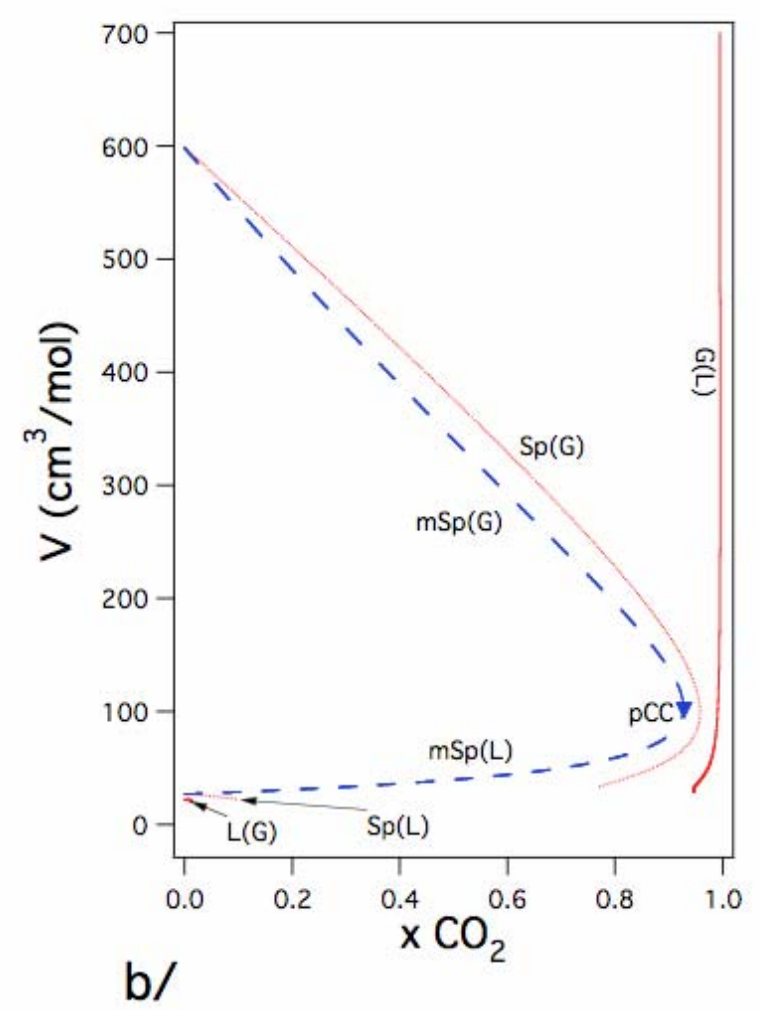

Figure 3 


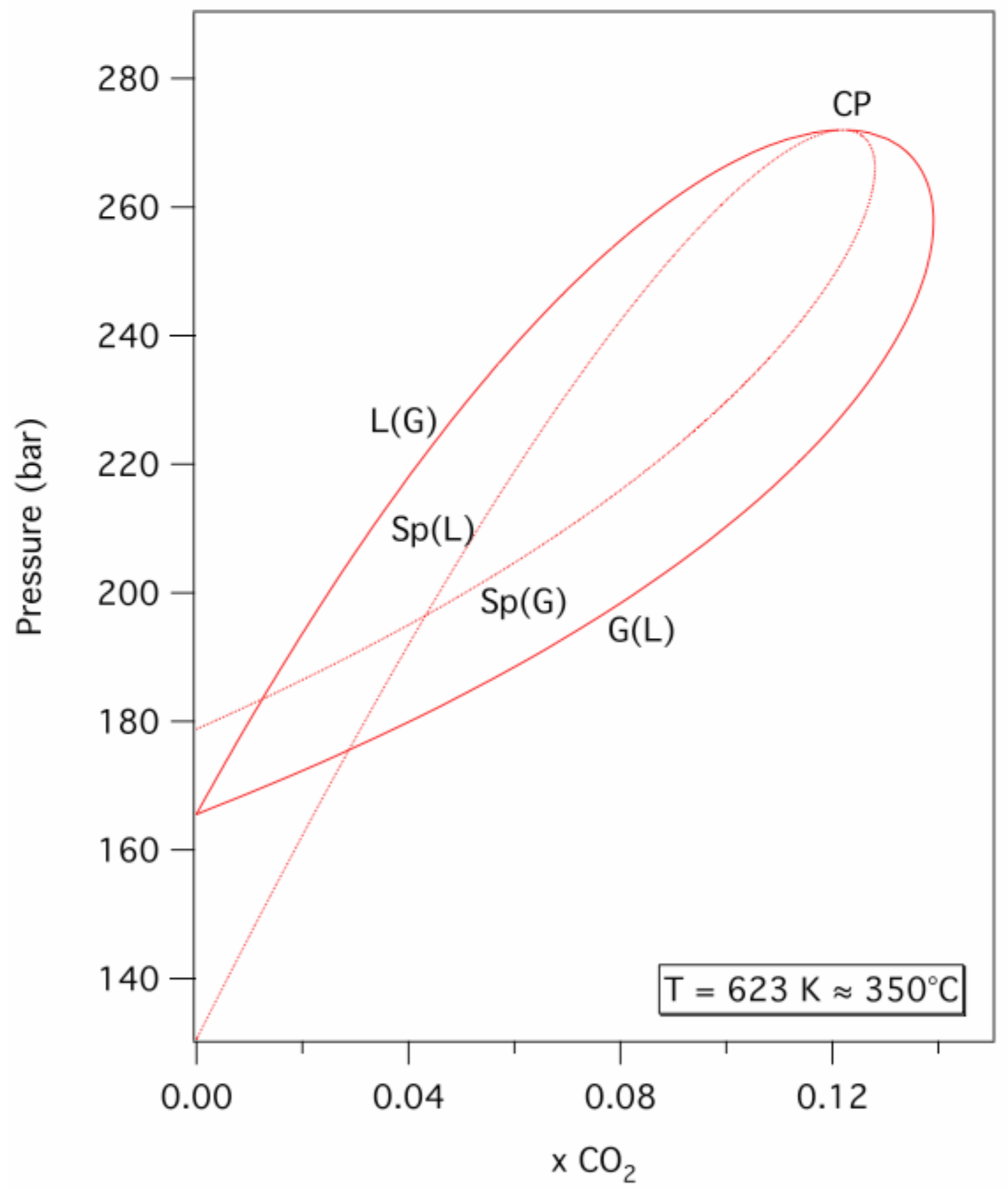

Figure 4 


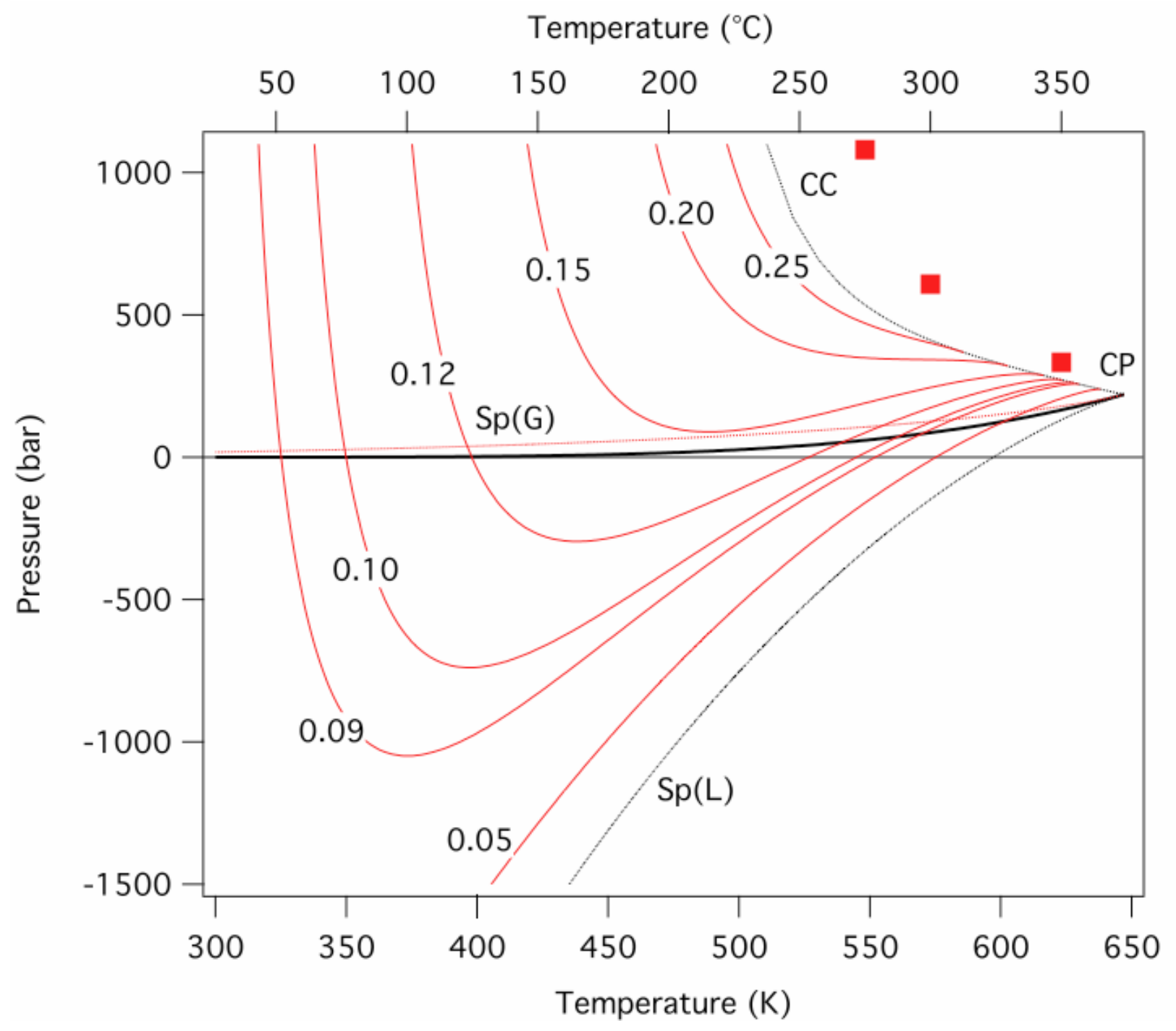

Figure 5 


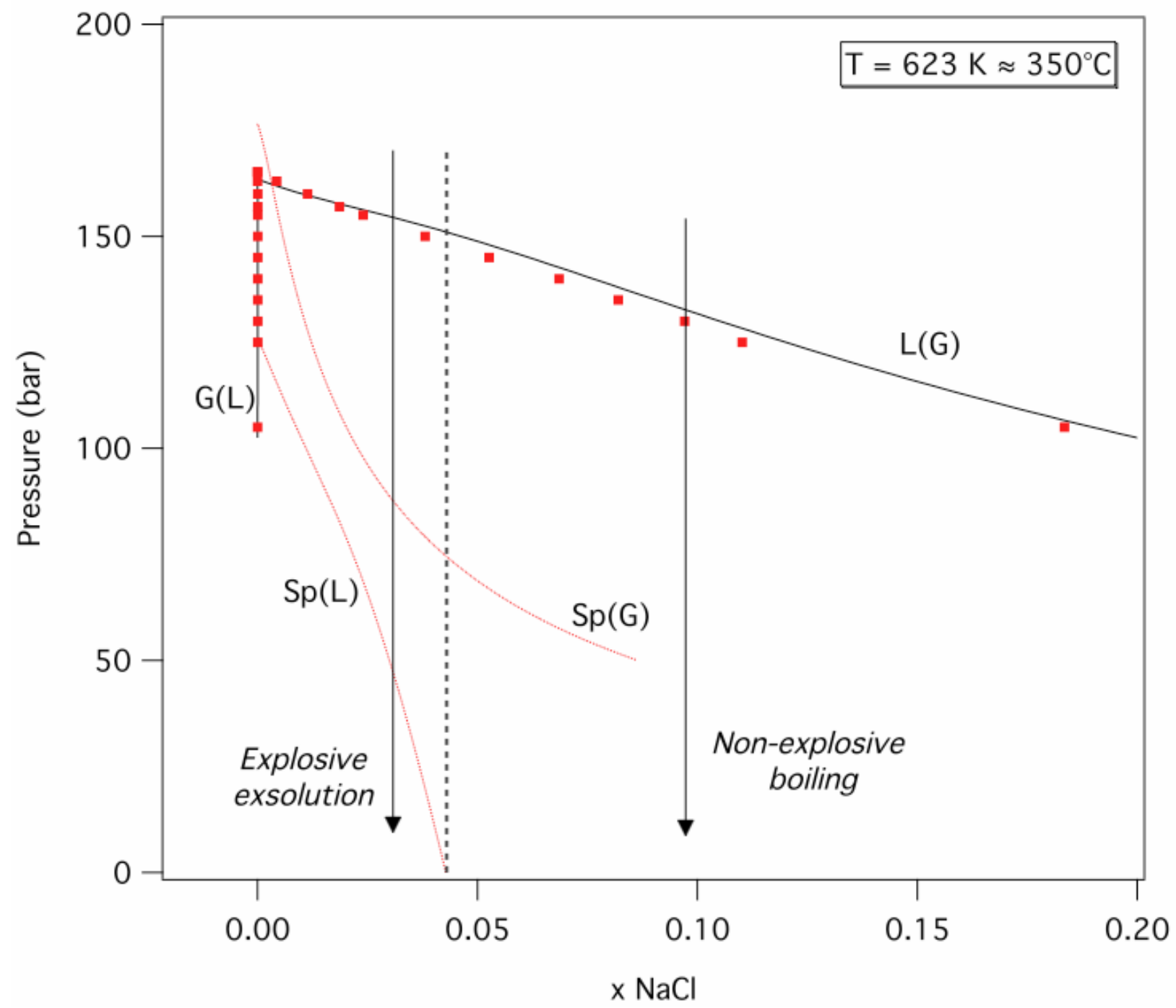

Figure 6 

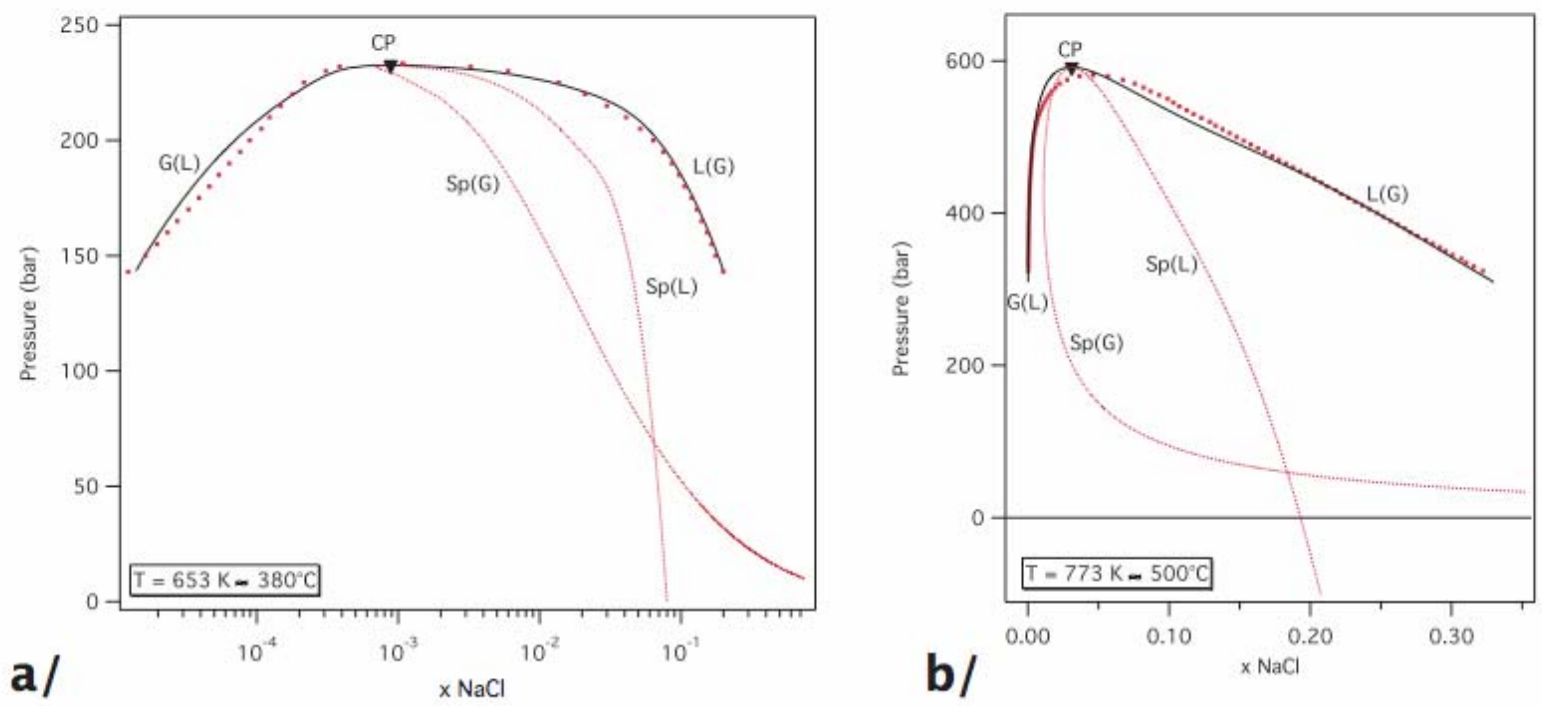

Figure 7 


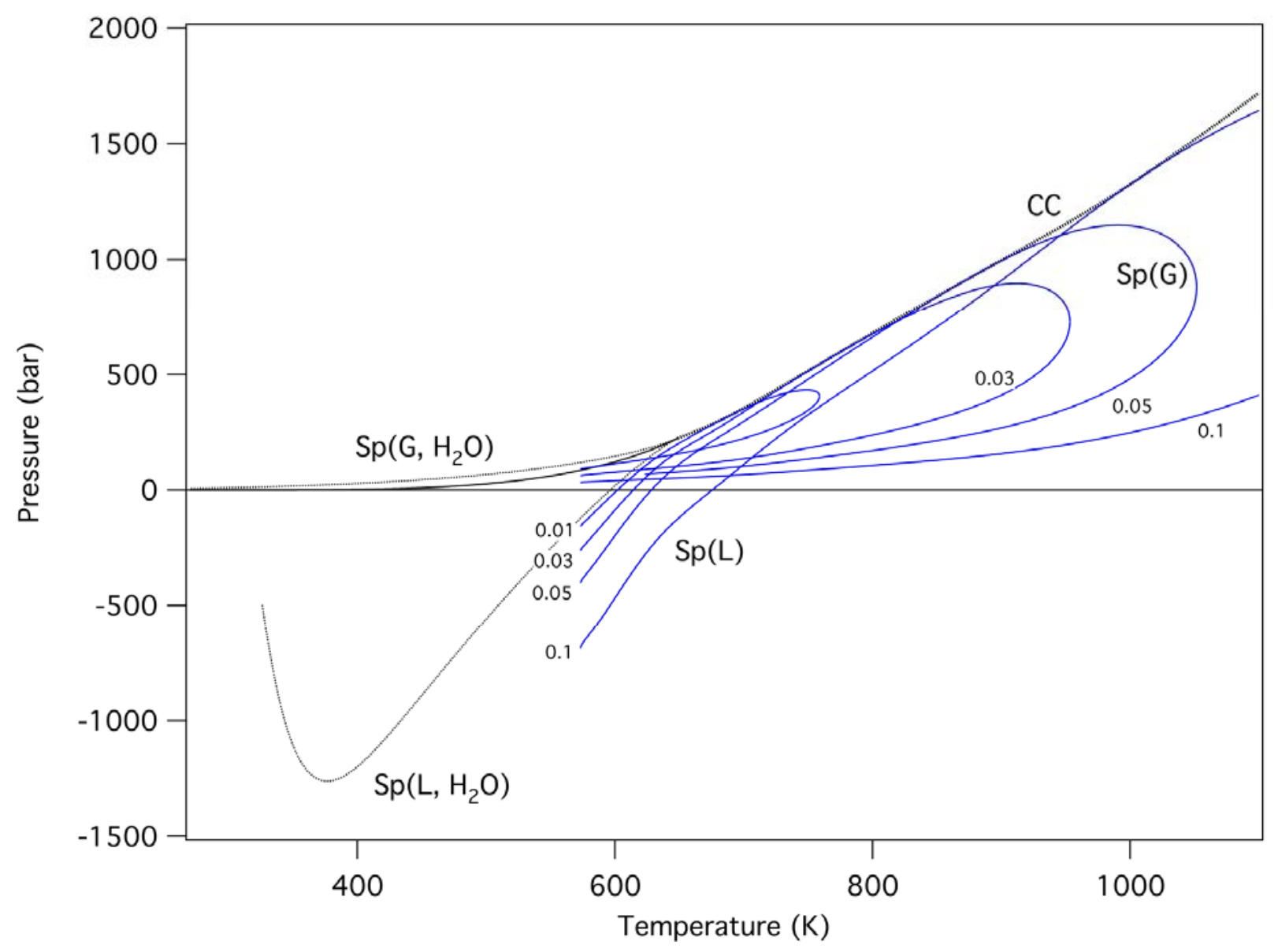

Figure 8 